\title{
Performance evaluation of optical attocells configuration in an indoor visible light communication
}

\author{
M S M Gismalla, M F L Abdullah \\ Department of Communication Engineering, Faculty of Electrical and Electronic Engineering, \\ Universiti Tun Hussein Onn Malaysia (UTHM), Malaysia
}

\begin{tabular}{l} 
Article Info \\
\hline Article history: \\
Received Sep 27, 2018 \\
Revised Nov 24, 2018 \\
Accepted Jan 18, 2019 \\
\hline
\end{tabular}

Keywords:

Optical attocells

Optical power distribution

Signal-to-noise ratio (SNR)

Visible light communication

Light emitted diodes (LED)

\begin{abstract}
Visible light communication VLC is deemed as futuristic technology applied for both illumination and data communication due to the low-cost energy consumption, long life expectancy, huge bandwidth, and high security compared to radio frequency RF. Uncovered area, minimum signal-to-noise ratio (SNR) and received power results from the non-uniform distribution of small base stations (i.e. Optical attocells) in the room. In this paper, the researchers propose a new LEDs distribution of five optical attocells configuration model in order to optimize the received power distribution and SNR at the center of the room for indoor VLC system. The optical attocells configuration in terms of received power to fill the uncovered area at the center of the room has been investigated. The simulation results showed that the proposed attocells configuration saved $24.9 \%$ of the transmitted power. Besides that, the whole room was covered uniformly. As a result, the received power and SNR are improved.
\end{abstract}

Copyright $(0) 2019$ Institute of Advanced Engineering and Science. All rights reserved.

\section{Corresponding Author:}

MSM Gismalla,

Department of Communication Engineering, Universiti Tun Hussein Onn Malaysia (UTHM),

Parit Raja, 86400 Batu Pahat, Johor, Malaysia.

Email: he160034@siswa.uthm.edu.my

\section{INTRODUCTION}

The evolution of solid-state lighting technologies open doors for researchers in both academia and industry fields to apply the LEDs devices in several valuable applications due to the unlimited free spectrum, low cost of energy consumption, long life expectancy, and security issue features. The current advances and technical challenges of LEDs were investigated in order to improve the performance of illumination [1]. The optical band was proposed as a supplementary technology to RF in the indoor application to transfer data with high rates through the LEDs. The optical band contains an infrared (IR), visible light (VL) and ultraviolet (UV) sub-bands. On February 11th of 1800, W. Herschel discovered the Infrared light, he discussed the spectrum of sunlight with the prism and determined the temperature of all colors [2]. The IR is used in short-range applications to transfer information with standard data rates ranged between $9.6 \mathrm{Kbps}$ and $16 \mathrm{Mbps}$ [3]. The UV radiation and its properties were discovered earlier in phases by three fellow scientists gradually in 1614, 1777, 1801 named Sala, Scheele, and Ritter respectively [4]. Visible light is unique in nature than IR and UV as known previously. The first experiment was achieved by Graham Bell in 1880 through an advanced a photo phone system that transmitted speech wirelessly through the modulation of reflected sunlight [5], [6]. Besides that, VLC offers high data rate that goes up to Gigabit/second by applying multicarrier modulation systems such as OFDM. The VLC wavelength has a range of 400-700 nm as demonstrated on electromagnetic spectrum shown in Figure 1, different LEDs are used to create a secure zone in the physical layer of a VLC system in order to provide a higher transmission security [7], [8]. In addition, the white LEDs lighting system of high radiated power produces an excellent quality of services [9]. 


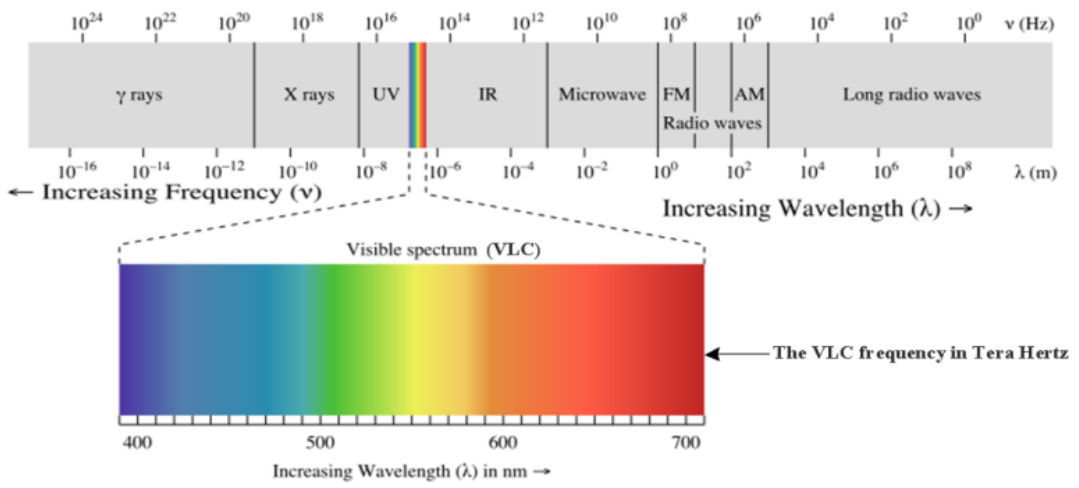

Figure 1. Frequency and wavelength of the electromagnetic spectrum [2]

\section{RELATED WORKS}

The VLC is in demand to realize the optical attocells network and become a new path towards the smaller cells [10]. Besides, the VLC system of multiple LED lighting was presented in an indoor ecosystem to connect several devices in short-range distances [11]. The visible light of the indoor transceiver system was investigated extensively. It gives the Li-Fi concept when the devices use IR or RF for uplink connection and light emitting diodes for the downlink connection. A new device allocation system of Li-Fi was suggested for multi-user in the transceiver system, the results of this suggestion produced a high performance and minimum interference in the VLC physical link [12]. The discussion of LEDs characteristics as illumination tools is required to control the emitted optical power in the room that participates in providing a minimum SNR to guarantee the data transmission between devices, besides, the enhancement of the quality of VLC system [13]. Until now the regularity of SNR in every location inside the indoor VL environment is obstructed by the multipath dispersion in the channel. The evolutionary algorithm (EA) was designed to optimize the dynamic SNR allocation in the room with dimensions same as [14] by using three light configuration design $\mathrm{A}, \mathrm{B}$ and $\mathrm{C}$ of $6 \times 6,7 \times 7$ and $5 \times 5$ LEDs arrays respectively, for the room, this approach was authenticated using tools with the ability to adjust the emitted power from each LED. The spectral efficiency of the cellular network increase when the cell sites are reduced, small cell notion of cellular system can be readily extended to VLC beating the high interference resulted from the proximate reuse of RF spectrum. Besides that, the optical attocell does not produce any extra interference, while improving indoor coverage. The light signal does not penetrate the walls due to the limited coverage zone of every single attocell that prevent the communication system from adjacent interference between neighbor rooms. These features facilitate applying manifold attocell to cover the whole room space. Figure 2 illustrated several cellular coverage zones, which included attocells configuration. The light distribution inside the room is imagined to work as tiny optical Base Station (BS), otherwise called the optical attocells. It is similar to the femtocell in the cellular communication system [15]. Besides that, the authors investigated the requirements of the $5 \mathrm{G}$ cellular system in terms of several parameters and various technologies to meet up to the expected performance demands.

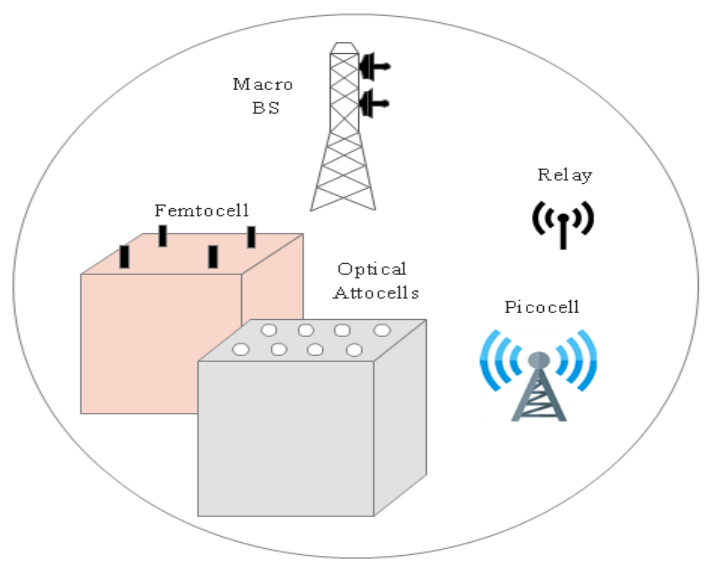

Figure 2. The attocell in the context of the heterogeneous network 
The LEDs distribution on the indoor environment was investigated to improve the average area spectral efficiency [16], the optimization was studied for two attocell on the room of $2.5 \mathrm{~m} \times 5 \mathrm{~m} \times 3 \mathrm{~m}$ dimension. MATLAB was used for simulation and the result shows that the average coverage area is maximized at high FOV.

In this paper, the authors study the performance of attocells configuration based on the coverage area. To the best of the researcher's knowledge, the proposed design is a novel solution for an optimal coverage area by applying a new LEDs distribution in five attocells configuration model. This new configuration model increases the coverage area, enhances the SNR and optical power distribution. The rest of this paper is organized as follows: Section 2 presents related works of VLC system. Section 3 is the system model, it involves two subsections, light propagation model of LOS channel, mathematical equations of the received power and SNR analysis. Section 4 presents the results analysis and discussion. The conclusion of this work is illustrated in Section 5.

\section{SYSTEM MODEL}

\subsection{Light Propagation Model}

In this paper, only the LOS propagation model for optimizing the indoor VLC environment as depicted in Figure 3 was considered. The light is distributed from the ceiling to the whole room and the Lambertian radiation pattern is considered for the source of emission [17]. The illumination intensity of angle $\phi$ is defined by:

$$
I(\phi)=I(0) \cos ^{m}(\phi)
$$

Where I (0) represent the center luminous intensity from a group of LEDs, $\phi$, and $\mathrm{m}$ represents the angle of irradiance and order of Lambertian emission respectively. In [18], The Lambertian radiant intensity is given by:

$$
R(\phi)=\frac{(m+1)}{2 \pi} \cos ^{m}(\phi)
$$

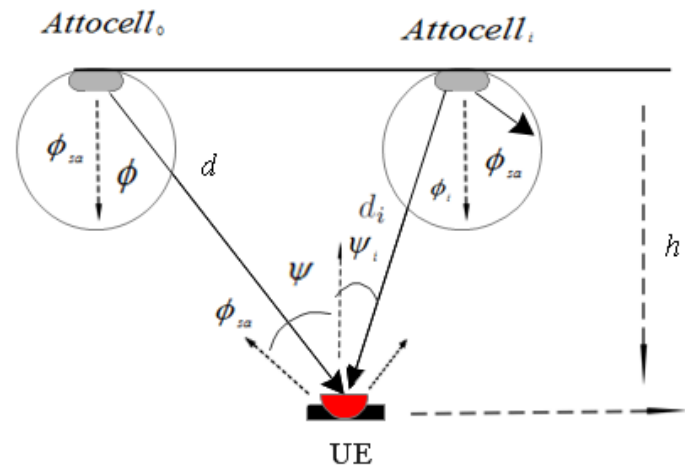

Figure 3. Geometry of LOS propagation model

The Lambertian coefficient is defined by semi-angle of half illumination from LEDs $\phi_{s a_{\frac{1}{2}}}$ such as:

$$
m=\frac{-\ln 2}{\ln \left(\cos \phi_{s a_{\frac{1}{2}}}\right)}
$$

The total transmitted power generated from the LEDs emission is given by:

$$
P=P_{L E D} * R(\phi)
$$

The VLC channel is described by LOS link and characterized by DC gain as illustrated in (5) 


$$
H_{L O S}=\left\{\begin{array}{c}
\frac{A_{r x}}{h^{2}} R(\phi) \cos (\varphi) 0 \leq \varphi \leq \varphi_{c} \\
0 \varphi>\varphi_{c}
\end{array}\right.
$$

Where $R(\phi)$ is defined in (2), $A_{r x}$ is the detector physical area of a photodiode (PD), $h^{2}$ is the distance between transmitter and receiver, $\varphi$ refers to the incident angle of the receiver, $\varphi_{c}$ is the field of view angle of PD. The whole power of LOS link can be computed using (6).

$$
P_{L O S}=\sum_{i=1}^{L E D_{\text {num }}} P * H_{L O S}
$$

The proposed optical attocells configuration is displayed in Figure 4. It is mounted on the ceiling, and the semi-angle at half power was controlled to avoid the co-channel interference.

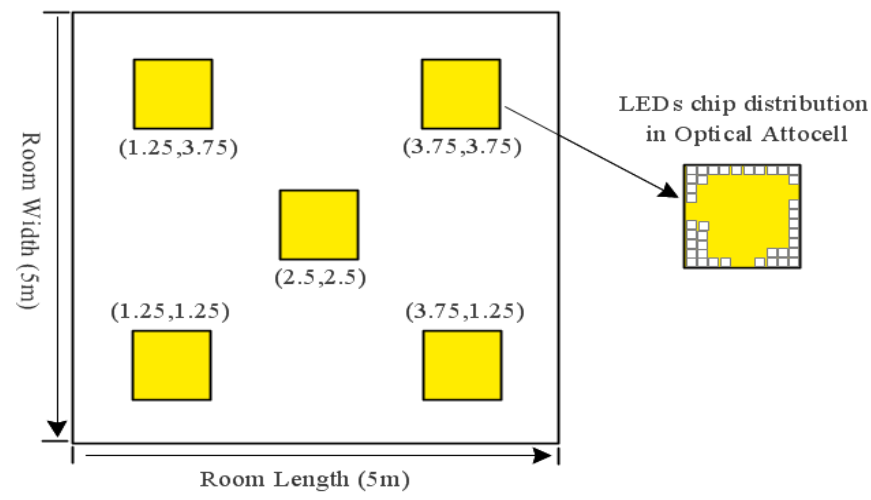

Figure 4. LEDs distribution in the proposed optical attocells network

\subsection{SNR Analysis}

Every communication system contains three basic parts transmitter, channel, and receiver. The channel of each communication system suffered from both internal and external random noise and distortion of the environment. The internal noise is difficult to remove in comparison to external noise which could be reduced and mitigated through various methods and mechanisms [19], [20]. In the source of the communication system, the transmitted intensity signal is sinusoid signal $\mathrm{x}(\mathrm{t})$ defined by an expression:

$$
x(t)=P_{t}(1+m \sin w t)
$$

Where $P_{t}$ represents the average transmitted power, and $\mathrm{m}$ define the amplitude of a sinusoidal signal. The received optical signal at the detector is represented by $\mathrm{r}(\mathrm{t})$, and it is defined by:

$$
r(t)=P_{r}(1+m \sin w t)+P_{a m b}
$$

Where $P_{r}$ represents the average power at the receiver, and $P_{a m b}$ represents the power of ambient light incident in the photodiode detector. The photodiode transforms the received optical intensity of the incident signal into photocurrent in accordance with the law of responsivity given below:

$$
R_{p}=\frac{I_{P}}{P_{P}}=\frac{q \eta_{i}}{h v}
$$

The responsivity $R_{P}$ is measured in amperes per watt, and it's defined as optoelectronic conversion factor that applied to convert the optical signal to the electrical domain. The received signal and DC measures of photocurrent can be separated in the following expression:

$$
\begin{gathered}
i_{\text {photo }}(t)=R_{p} \cdot r(t) \\
=R_{p}\left(P_{r}+P_{a m b}\right)+\left(R_{p} P_{r} m \sin w t\right)
\end{gathered}
$$

The power of an electrical signal at the receiver is given by: 


$$
P_{\text {signal }}=\frac{1}{2} m^{2} R_{p} P_{r}^{2}
$$

The photo-generated shot noise increases at the receiver due to the transmitted signal and the ambient light. Subsequently, the received photocurrent considers only a DC component at condition $P_{a m b} \gg$ $P_{r}$. Also, the noise power results from the pre-amplifier $\overline{l_{\text {curc }}^{2}}$ and shot noise $\overline{l_{\text {shot }}^{2}}$ are uncorrelated and the total noise power can be simply expressed by:

$$
\begin{gathered}
P_{\text {noise }}=\overline{l_{\text {crrc }}^{2}}+\overline{l_{\text {shot }}^{2}} \\
=\overline{l_{\text {crrc }}^{2}}+2 q R_{p}\left(P_{r}+P_{\text {amb }}\right) \cdot B_{\text {eff }}
\end{gathered}
$$

The $B_{\text {eff }}$ represents the equivalent noise bandwidth of the system, $\mathrm{q}$ is the electronic charge. From (11) and (12), the signal to noise ratio (SNR) is approximately expressed by:

$$
S N R_{\text {link }}=\frac{P_{\text {signal }}}{P_{\text {noise }}}
$$

The next section shows the simulations results and analysis; it was achieved based on the parameters illustrated in Table 1.

Table 1. Parameters of Simulation

\begin{tabular}{lc}
\hline \multicolumn{1}{c}{ Parameters } & Value \\
\hline Room dimension in meter & $5 \times 5 \times 3 \mathrm{~m}$ \\
Transmitted optical power by individual LED & $20 \mathrm{~mW}$ \\
Total transmitted power (single attocell) & $54 \mathrm{~W}$ \\
Detector physical area (PD) & $1 \mathrm{~cm}^{2}$ \\
Gain of an optical filter (Ts) & 1 \\
Refractive index & 1.5 \\
FOV of a receiver & $60^{\circ}$ \\
LED center luminous intensity & 0.73 \\
Semi-angle at half power & $0.5^{\circ}, 20^{\circ}, 30^{\circ}, 40^{\circ}, 50^{\circ}, 60^{\circ}, 70^{\circ}, 80^{\circ}$ \\
Table height & $0.85 \mathrm{~m}$ \\
Transmitter position (Attocells) & 4 Attocells: $^{\circ}(1.25,1.25),(1.25,3.75)$, \\
& $(3.75,1.25),(3.75,3.75)$ \\
& 5 Attocells: $(1.25,1.25),(1.25,3.75)$, \\
Responsivity of PD & $(2.5,2.5),(3.75,1.25),(3.75,3.75)$ \\
Bandwidth & $0.54(\mathrm{~A} / \mathrm{W})$ \\
Temperature & $30 \mathrm{Mb} / \mathrm{s}$ \\
\end{tabular}

\section{RESULTS AND ANALYSIS}

The performance evaluation of multiple attocells configuration was measured as shown in Table 2. The amount of optical received power in five locations in the room as represented by $(1.25,1.25)$, $(1.25,3.75),(2.5,2.5),(3.75,1.25),(3.75,3.75)$ were investigated. In a typical four attocells configuration, the maximum received power is concentrated at the corners of the room. The center of the room received low optical power and SNR distribution. Interestingly, the new LEDs distribution of optical attocells configuration has been producing a uniform power and SNR distribution in the whole room. In this manner,

\begin{tabular}{|c|c|c|c|c|c|}
\hline \multirow{2}{*}{$\begin{array}{c}\text { Optical Attocells } \\
\text { configuration with } \\
\text { different semi-angle } \varphi_{1}\end{array}$} & \multicolumn{5}{|c|}{ Optical Received Power at different positions (dBm) } \\
\hline & $(1.25,3.75)$ & $(1.25,1.25)$ & $(2.5,2.5)$ & $(3.75,1.25)$ & $(3.75,3.75)$ \\
\hline Four & 12.76 & 12.76 & -16.99 & 12.76 & 12.76 \\
\hline Five & 12.23 & 12.23 & 12.23 & 12.23 & 12.23 \\
\hline Four & 1.966 & 1.966 & 2.101 & 1.966 & 1.966 \\
\hline Five & 2.429 & 2.429 & 3.718 & 2.429 & 2.429 \\
\hline
\end{tabular}
the proposed configuration model consumes less transmission power than that applied in four attocells configuration.

Table 2. Received Power of Different Attocells Configuration 
Figure 5 to Figure 8 shows the received power and SNR distribution at semi-angle at half power $12.5^{\circ}$ and $70^{\circ}$ respectively. There is a trade between the received power and SNR distribution. At small semiangle at half power, the received power is increased and SNR is decreased, and when the semi-angle at half power is increased, the SNR distribution increased and the received power will be decreased. Clearly, the results were improved as compared to [21] [22].

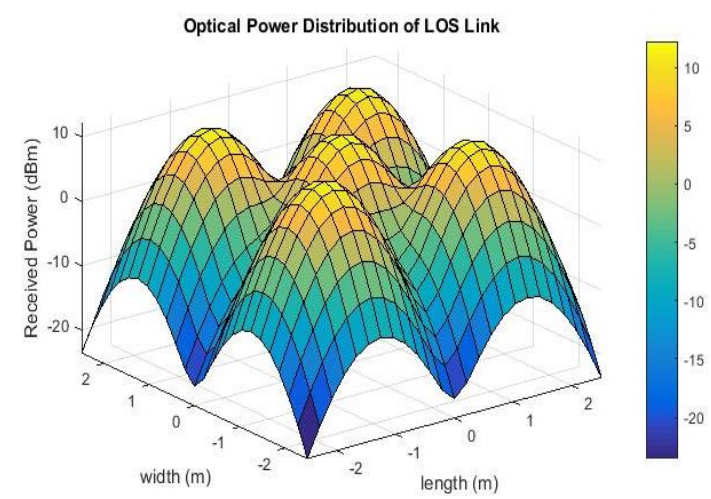

Figure 5. Received optical power $\left(12.5^{\circ}\right)$

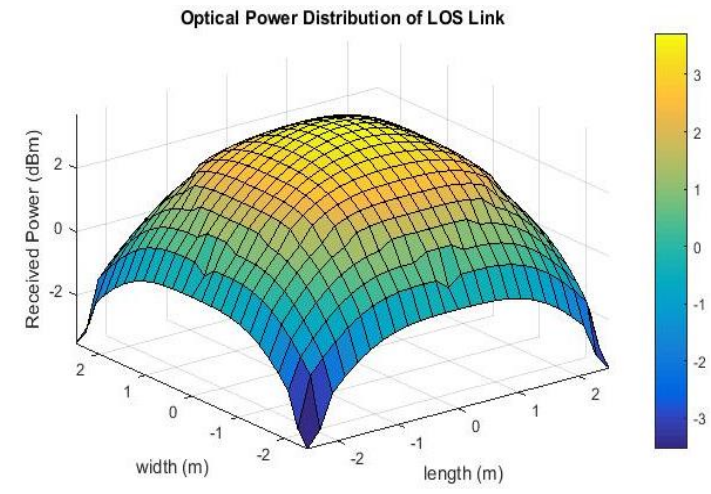

Figure 7. Received optical power $\left(70^{\circ}\right)$

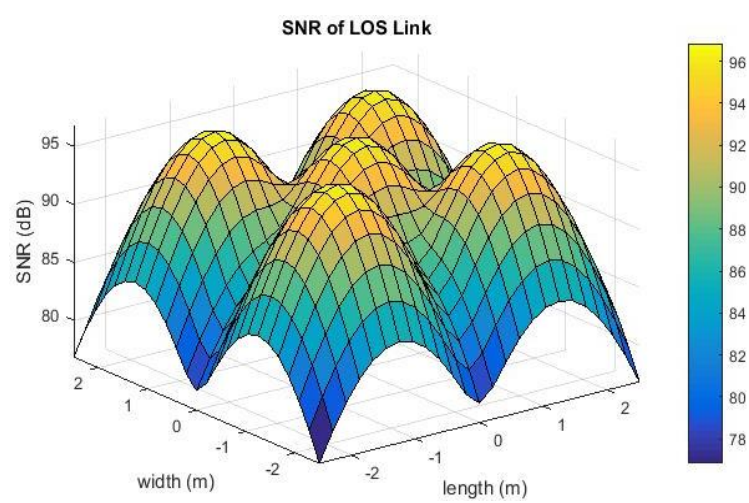

Figure 6: SNR distribution $\left(12.5^{\circ}\right)$

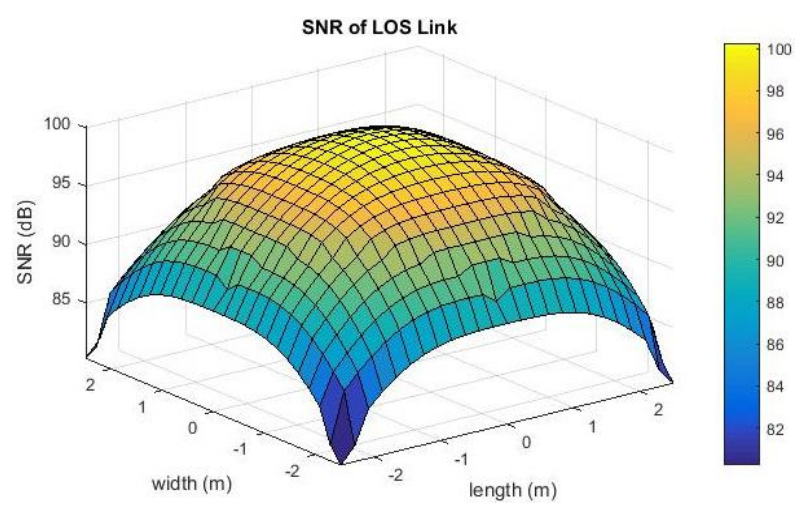

Figure 8: SNR distribution $\left(70^{\circ}\right)$

Figure 9 shows the coverage area of the room in terms of optical received power for the four attocells configurations; the authors observed a small power distribution at the center of the room. To avoid this problem, the five attocells configuration with less radiated power has been proposed as demonstrated in Figure 10.

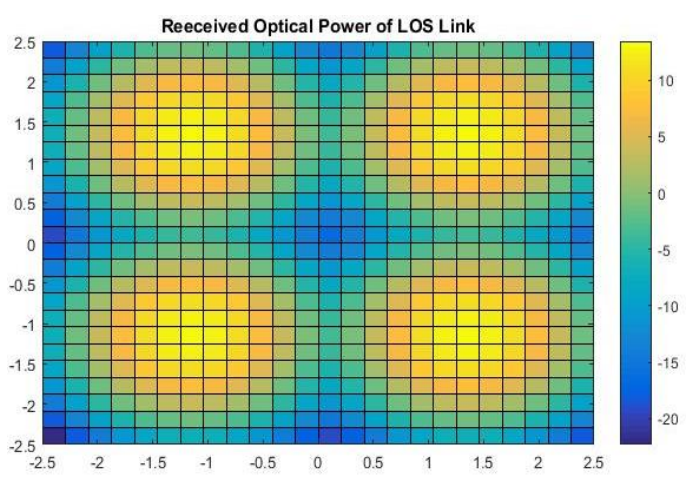

Figure 9. Coverage area of four attocells $\left(12.5^{\circ}\right)$

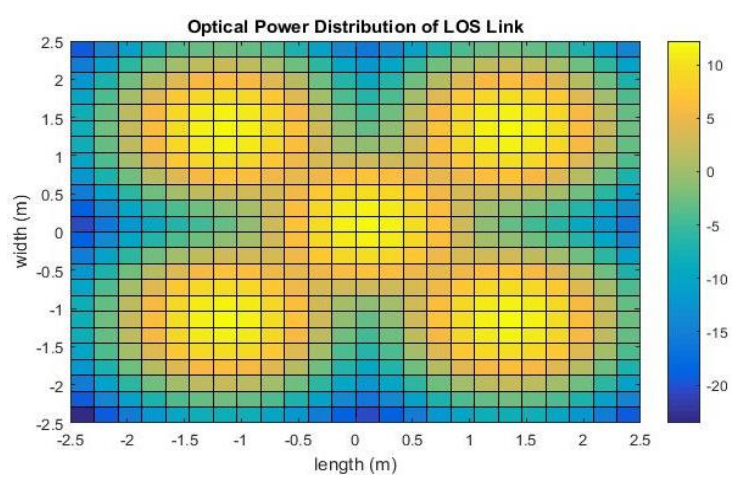

Figure 10. Coverage area of five attocells $\left(12.5^{\circ}\right)$ 
The results showed that the SNR and the received power at the center of the room were improved. Besides that, the four optical attocells configuration in previous studies was used 14400 LEDs chip while the proposed optical attocells configuration has used 13520 LEDs chip. Figure 11 and Figure 12 shows maximum optical received power at the center of ceiling for the various semi-angle at half power and field of view respectively. Figure 13 showed the total SNR distribution at the center of the room for all-optical attocells network. It was improved with an average difference ratio of $9.12 \%$. The simulation results showed that both total SNR and the optical received power were improved. Subsequently, the coverage area of the whole room was increased uniformly as compared to [23], [24].

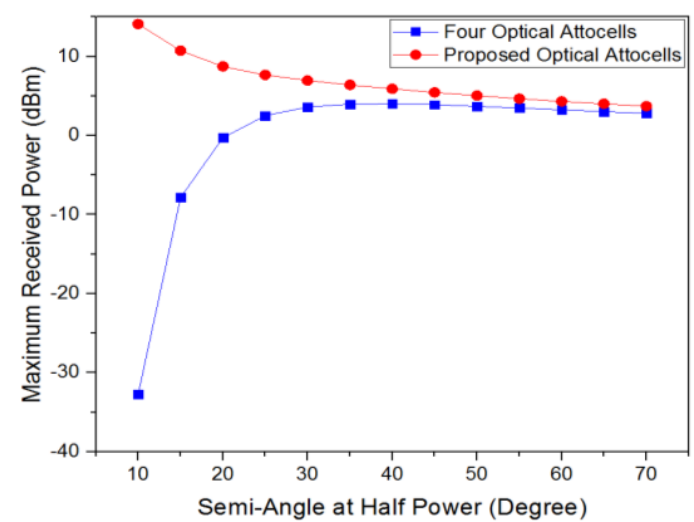

Figure 11. Maximum optical received power distribution at the center of the room

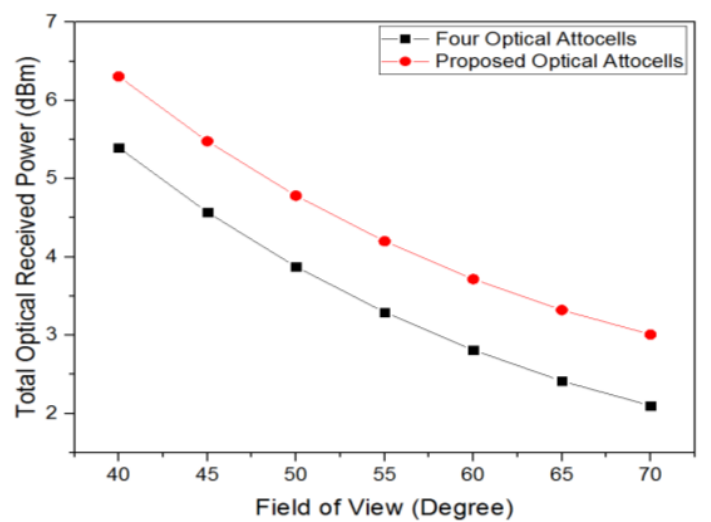

Figure 12. Total received power at the center of the room under various FOV

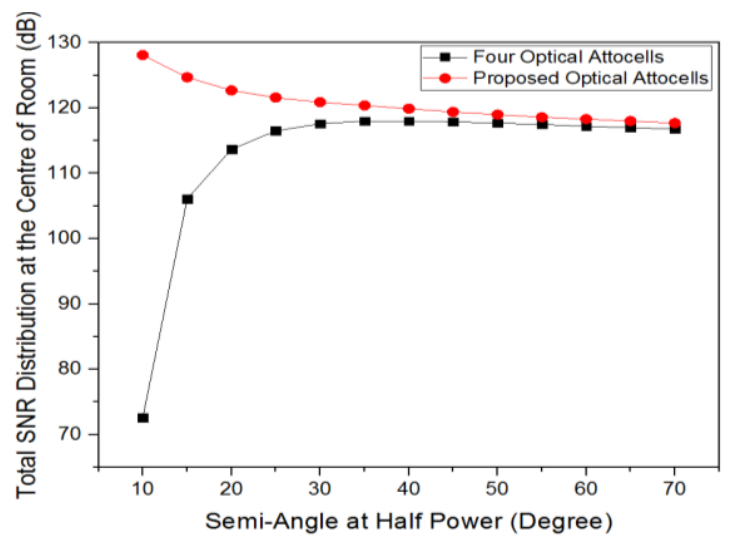

Figure 13. Total SNR distribution of all optical attocells network

\section{CONCLUSION}

Visible light communication is a new emerging technology alternative to radio frequency in the indoor communication systems. It's proposed as a promising and a powerful technology for $5 \mathrm{G}$ networks. In this paper, the authors investigated the optical attocells configuration in the room under the LOS condition to fill the uncovered area. Besides that, the SNR and optical received power were optimized by developing a new LEDs distribution of five attocells configuration. The simulation results of the proposed attocells configuration displayed that the optical received power against FOV has increased with an average difference ratio of $28 \%$. Also, the total SNR has improved with average difference ratio of $9.12 \%$. Subsequently, the coverage area was increased uniformly. In coming future work, the same attocells configuration will be applied to non-LOS propagation link, towards enhancing the link quality and performance of visible light communication systems. 


\section{ACKNOWLEDGEMENTS}

The authors of this paper wish to acknowledge the funding of this project by Center of Graduate Studies (CGS) Universiti Tun Hussein Onn Malaysia (UTHM).

\section{REFERENCES}

[1] M. H. Crawford, "LEDs for solid-state lighting: performance challenges and recent advances," IEEE J. Sel. Top. Quantum Electron., vol. 15, no. 4, pp. 1028-1040, 2009.

[2] M. Uysal and H. Nouri, "Optical wireless communications-An emerging technology," in Transparent Optical Networks (ICTON), 2014 16th International Conference on, 2014, pp. 1-7.

[3] D. K. Borah, A. C. Boucouvalas, C. C. Davis, S. Hranilovic, and K. Yiannopoulos, "A review of communicationoriented optical wireless systems," EURASIP J. Wirel. Commun. Netw., vol. 2012, no. 1, p. 91, 2012.

[4] C.-W. Chow, C.-Y. Chen, and S.-H. Chen, "Enhancement of signal performance in LED visible light communications using mobile phone camera," IEEE Photonics J., vol. 7, no. 5, pp. 1-7, 2015.

[5] L. Feng, R. Q. Hu, J. Wang, P. Xu, and Y. Qian, "Applying VLC in 5G Networks: Architectures and Key Technologies," IEEE Netw., vol. 30, no. 6, pp. 77-83, 2016.

[6] H. Elgala, R. Mesleh, and H. Haas, "Indoor optical wireless communication: Potential and state-of-the-art," IEEE Commun. Mag., vol. 49, no. 9, pp. 56-62, 2011.

[7] P. Kocharoen, "Visible Light Communication: Importance and Thai Preparations," Procedia Comput. Sci., vol. 86, pp. 51-54, 2016.

[8] C.-W. Chow, Y. Liu, C.-H. Yeh, C.-Y. Chen, C.-N. Lin, and D.-Z. Hsu, "Secure communication zone for whitelight LED visible light communication,” Opt. Commun., vol. 344, pp. 81-85, 2015.

[9] T. Komine and M. Nakagawa, "Integrated system of white LED visible-light communication and power-line communication," IEEE Trans. Consum. Electron., vol. 49, no. 1, pp. 71-79, 2003.

[10] D. Tsonev, S. Videv, and H. Haas, "Light fidelity (Li-Fi): towards all-optical networking," in Broadband Access Communication Technologies VIII, 2014, vol. 9007, p. 900702.

[11] K. Warmerdam, A. Pandharipande, and D. Caicedo, "Connectivity in IoT indoor lighting systems with visible light communications," in Green Communications (OnlineGreenComm), 2015 IEEE Online Conference on, 2015, pp. 47-52.

[12] A. Sewaiwar, S. V. Tiwari, and Y.-H. Chung, "Novel user allocation scheme for full duplex multiuser bidirectional Li-Fi network," Opt. Commun., vol. 339, pp. 153-156, 2015.

[13] Z. Wang, C. Yu, W.-D. Zhong, J. Chen, and W. Chen, "Performance of a novel LED lamp arrangement to reduce SNR fluctuation for multi-user visible light communication systems," Opt. Express, vol. 20, no. 4, pp. 4564-4573, 2012.

[14] C. Gong, S. Li, Q. Gao, and Z. Xu, "Power and rate optimization for visible light communication system with lighting constraints," IEEE Trans. signal Process., vol. 63, no. 16, pp. 4245-4256, 2015.

[15] C.-X. Wang et al., "Cellular architecture and key technologies for $5 \mathrm{G}$ wireless communication networks," IEEE Commun. Mag., vol. 52, no. 2, pp. 122-130, 2014.

[16] I. Stefan and H. Haas, "Analysis of optimal placement of LED arrays for visible light communication," in Vehicular Technology Conference (VTC Spring), 2013 IEEE 77th, 2013, pp. 1-5.

[17] H. Q. Nguyen et al., "A MATLAB-based simulation program for indoor visible light communication system," Commun. Syst. Networks Digit. Signal Process. (CSNDSP), 2010 7th Int. Symp., vol. 3600, no. April 2016, pp. 537-541, 2010.

[18] T. H. Do and M. Yoo, "Received power and SNR optimization for visible light communication system," ICUFN 2012 - 4th Int. Conf. Ubiquitous Futur. Networks, Final Progr., no. 2010, pp. 6-7, 2012.

[19] S. Hranilovic, Wireless Optical Communication Systems. 2005.

[20] Z. Ghassemlooy, L. N. Alves, S. Zvanovec, and M.-A. Khalighi, Visible Light Communications: Theory and Applications. CRC Press, 2017.

[21] Z. Ghassemlooy, W. Popoola, and S. Rajbhandari, Optical wireless communications: system and channel modelling with Matlabß. CRC press, 2012.

[22] T. Ganjian, G. Baghersalimi, and Z. Ghassemlooy, "Performance evaluation of the received power based on the transmitter position in a visible light communications system," 2017 Iran. Conf. Electr. Eng., no. May, pp. 1763-1768, 2017.

[23] J. H. Liu, Q. Li, and X. Y. Zhang, "Cellular coverage optimization for indoor visible light communication and illumination networks,” J. Commun., vol. 9, no. 11, pp. 891-898, 2014.

[24] H. Lu, Z. Su, and B. Yuan, "SNR and optical power distribution in an indoor visible light communication system," in Image and Signal Processing (CISP), 2014 7th International Congress on, 2014, pp. 1063-1067. 


\section{BIOGRAPHIES OF AUTHORS}

Mohammed Salih Mohammed Gismalla, received B.Sc (Hons) in Electronic and Electrical
Engineering (Communication) from the International University of Africa (IUA), Sudan in
2010, and M.Sc in Electronic Engineering (Communication) from Sudan University of
Science and Technology in 2015. He worked as teaching assistant in the Department of
Electronic and Electrical Engineering, Faculty of Engineering at IUA in 2010 and promoted
to Lecturer in 2015. He is working on his Ph.D. degree at the Universiti Tun Hussein Onn
Malaysia (UTHM), Malaysia. His research interests are focused on Visible Light
Communication and D2D communication.

